\title{
FINITE SPEED PROPAGATION IN THE RELAXATION OF VORTEX PATCHES
}

\author{
$\mathrm{Br}$ \\ CAROLE ROSIER (Analyse Numérique, Université Lyon 1, bât. 101, 69622 \\ Villeurbanne Cedex, France) \\ ANI) \\ LIONEL ROSIER (Institut Elie Cartan, B.P. 239, 54506 Vandouvre lès Nancy Cedex, France)
}

The paper is dedicated to the memory of Louis-Hubert Rosier.

\begin{abstract}
A degenerate parabolic equation has been proposed by Robert and Sommeria to describe the relaxation towards a statistical equilibrium state for a two-dimensional incompressible perfect fluid with a vortex patch as initial vorticity. In this paper, flows obtained by numerical integration of the Robert-Sommeria equation over a long-time interval are compared with those obtained for the Navier-Stokes equation at high Reynolds number. A finite speed propagation for the extremal values of the vorticity is numerically shown to hold for the Robert-Sommeria equation. A rigorous proof of this (fine) property is also provided.
\end{abstract}

1. Introduction. The motion of a two-dimensional incompressible perfect fluid is described by the Euler equation which, in the classical stream function-vorticity formulation, reads as follows:

$$
\frac{\partial \omega}{\partial t}+\operatorname{div}(\omega \nabla \times \psi)=0, \quad t \geq 0, y \in \mathcal{Y} .
$$

Here $t$ is time, $\mathcal{Y}$ denotes a bounded domain in $\mathbb{R}^{2}, \omega=\omega(t, y)$ is the vorticity and $\psi$ ' is the corresponding stream function, obtained by solving the Laplace equation $-\Delta \psi=\omega$ in $\mathcal{Y}$ together with some appropriate boundary conditions. As time proceeds, the vorticity exhibits rapid, local fluctuations at smaller and smaller scales, whereas large scale structures (the so-called coherent structures) seem to cmerge as $t \rightarrow+\infty$. A statistical equilibrium theory has been developed by Miller et al. [11] [12] and Robert et al. [14] [15] to explain the emergence of these coherent structures. According to this theory, a

Received June 19, 2000 and, in revised form. April 10, 2001.

2000 Mathematics Subject Classification. Primary 35K65, 35Q35, 76C05, 76F99.

Key words and phrases. Relaxation equation, Navier-Stokes equation, statistical equilibrium, finite speed propagation.

E-mail address: rosier@lan.univ-lyon1.fr

E-mail address: rosier@antares.iecn.u-nancy.fr 
coherent structure is identified with a statistical equilibrium state, characterized by maximizing mixing entropy subject to constraints dictated by the conservation of circulation, kinetic energy and generalized enstrophies (and also angular impulse, when $\mathcal{Y}$ is a ball). An efficient numerical method for solving the constrained maximum entropy problem has been presented by Turkington et al. in [20]- [21]. Their predictions based on statistical equilibrium computations are consistent with the conclusions drawn from direct simulations of high Reynolds" flows, at least for regimes with a high rate of relaxation into equilibrium. In regimes with gradual relaxation, however, the computed statistical equilibrium state may fail to emerge over the time interval of the computed evolution. Several reasons may be invoked [21]: (i) there is a gap between the (long) time scale needed for the relaxation and the (short) time scale over which direct simulations are performed, or (ii) the accumulated effects of small-scale vorticity fluctuations (such as filamentation) are lost by truncation in the direct simulation, or (iii) the equilibrium state obtained when integrating the Navier-Stokes equation (instead of the Euler equation) over a longtime interval is deeply altered by energy dissipation. A macroscopic evolution equation, whose numerical integration over a sufficiently long time interval always leads to the statistical equilibrium state, has been proposed by Robert and Sommeria in [16]. This relaxation equation is a degenerate parabolic equation of diffusion-convection type. It has been designed to conserve above constraints (in particular, the kinetic energy) and to maximize at each time the production of entropy. It may serve as a model for the dynamics of the macroscopic vorticity $\bar{\omega}$ obtained from $\omega$ by taking local mean value, i.e.. $\bar{\omega}\left(y_{0}\right):=\lim _{r \rightarrow 0} \pi^{-1} r^{-2} \int_{B_{r}\left(y_{0}\right)} \omega(y) d y$ for any $y_{0} \in \mathcal{Y}$. Simulating Robert-Sommeria's relaxation equation is particularly interesting, since it provides at the same time (i) the (exact) statistical equilibrium state (over a relatively short time scale, when compared with Navier-Stokes simulations), and (ii) a coarse-grained description of the relaxation process towards the equilibrium state.

From now on, for computational convenience, we limit ourselves to the case where $\mathcal{Y}$ is a periodic domain, i.e., $\mathcal{Y}=(\mathbb{R} / \mathbb{Z})^{2}=\left(-\frac{1}{2}, \frac{1}{2}\right)^{2}$ with periodic boundary conditions in $y_{1}$ and $y_{2}$, and we assume that the initial vorticity $\omega_{0}$ is made up of patches with $n$ uniform vorticity levels $a_{i}$. (To be brief, we shall say that $\omega_{0}$ is a vortex patch.) The macroscopic vorticity $\bar{\omega}$ may be described in terms of a set of local probabilities $p_{1}(t, y) \ldots p_{n}(t, y)$ as follows:

$$
\bar{\omega}(t, y)=\sum_{i=1}^{n} a_{i} p_{i}(t, y) .
$$

Here, $p_{i}(t, y) \geq 0$ for each $i$ and $\sum_{i} p_{i}(t, y)=1$, for all pairs $(t, y)$. The stream function $\bar{\psi}$ associated with $\bar{\omega}$ is defined by

$$
\left\{\begin{array}{l}
-\Delta \bar{\psi}=\bar{\omega} \text { in } \mathcal{Y} . \\
\bar{\psi} \text { is 1-periodic in } y_{1}, y_{2} \text { and } \int_{\mathcal{Y}} \bar{\psi} d y=0 .
\end{array}\right.
$$

(Notice that the condition $\int_{\mathcal{Y}} \bar{\omega}(y) d y=0$ has to be satisfied in order that $\bar{\psi}$ be welldefined.) $\nabla \times \psi=\left(\partial \psi / \partial y_{2},-\partial \psi / \partial y_{1}\right)$ is the corresponding velocity field. Set $\overline{\omega^{2}}:=$ 
$\sum_{i} a_{i}^{2} p_{i}, A:=\nu\left(\overline{\omega^{2}}-\bar{\omega}^{2}\right)(\nu>0$ being some constant coefficient $)$ and

$$
\beta:=-\frac{\int_{\mathcal{Y}} \nabla \bar{\psi} \cdot \nabla \bar{\omega} A d y}{\int_{\mathcal{Y}}|\nabla \bar{\psi}|^{2}\left(\overline{\omega^{2}}-\bar{\omega}^{2}\right) A d y} .
$$

Then the relaxation equation associated with the vortex patch $\omega_{0}: \mathcal{Y} \rightarrow\left\{a_{1}, \ldots, a_{n}\right\}$ reads as follows (see [16], [17])

$$
(\operatorname{RE} n) \quad \frac{\partial p_{i}}{\partial t}+\operatorname{div}\left(p_{i} \nabla \times \bar{\psi}-A\left(\nabla p_{i}-\beta\left(\bar{\omega}-a_{i}\right) p_{i} \nabla \bar{\psi}\right)\right)=0, \quad i=1, \ldots, n .
$$

Each $p_{i}$ is 1-periodic in $y_{1}, y_{2}$ and it fulfills

$$
p_{i}(0, y)=\lim _{r \rightarrow 0} \frac{\lambda\left(\omega_{0}^{-1}\left(a_{i}\right) \cap B_{r}(y)\right)}{\lambda\left(B_{r}(y)\right)}
$$

for a.e. $y \in \mathcal{Y}$, where $\lambda$ denotes the Lebesgue measure. (Notice that $\int_{\mathcal{Y}} \bar{\omega}(0, y) d y=$ 0 whenever $\int_{\mathcal{Y}} \omega_{0}(y) d y=0$.) We emphasize that the $p_{i}$ 's (hence also $\bar{\omega}, \overline{\omega^{2}}, A$ and $\bar{\psi}$ ) are functions of $t$ and $y$, whereas the (inverse temperature) $\beta$ depends only on $t$. Since $\sum_{i} p_{i}=1$, there are in fact only $n-1$ unknown functions in (REn), namely $p_{1}, \ldots, p_{n-1}$. In this paper, attention will be focused on the simplest cases, namely (RE2) (with $a_{1}=1, a_{2}=-1$ ) and (RE3) (with $a_{1}>0, a_{2}<0, a_{3}=0$ ). When $n=2$, $\bar{\omega}=2 p_{1}-1(\in[-1,1])$; hence, (RE2) may be rewritten as

$$
\frac{\partial \bar{\omega}}{\partial t}-\nu \operatorname{div}\left(\left(1-\bar{\omega}^{2}\right) \nabla \bar{\omega}\right)+\operatorname{div}(\bar{\omega} \nabla \times \bar{\psi})-\nu \beta(\bar{\omega}) \operatorname{div}\left(\left(1-\bar{\omega}^{2}\right)^{2} \nabla \bar{\psi}\right)=0,
$$

with the following expression for $\beta$ obtained by integration by part

$$
\beta=-\frac{\int_{\mathcal{Y}} \bar{\omega}^{2}\left(1-\frac{\bar{\omega}^{2}}{3}\right) d y}{\int_{\mathcal{Y}}|\nabla \psi|^{2}\left(1-\bar{\omega}^{2}\right)^{2} d y} .
$$

A rather complete theory for (RE2), including local existence and uniqueness for any initial datum and global existence and asymptotic bchavior for a large class of initial data, has been carried out by the authors in [18]. This equation has not yet been numerically simulated, contrary to (RE3) [17]. In this paper, we first test the validity of this new model by means of numerical simulations compared with those using the NavierStokes equation at high Reynolds number. Both the transient relaxation processes and the final equilibrium states will be compared. Finally, the important issue of whether the vorticity remains unchanged in the core of a patch will be investigated. In contrast to what happens for the Navier-Stokes equation, a finite speed propagation for the extremal values (namely \pm 1 ) of the vorticity occurs for (RE2), due to the degenerate diffusion term $\operatorname{div}\left(\left(1-\bar{\omega}^{2}\right) \nabla \bar{\omega}\right)$. Given any patch where $\omega_{0} \equiv+1$ (resp. $\omega_{0} \equiv-1$ ), it means that the value +1 (resp. -1$)$ is still taken by $\bar{\omega}(t,$.$) in the core of the patch, at least for small$ enough times. Numerical simulations will demonstrate that this property indeed occurs for (RE2) (and that it fails to be true for the Navier-Stokes equation). A rigorous proof of the finite speed propagation property will also be provided, for the sake of completeness.

The paper is outlined as follows. In Sec. 2, the main results pertaining to (RE2), namely those in [18] and the finite speed propagation property (Theorem 2.4), are stated without proof for the reader's convenience. The proof of the finite speed propagation 
property is given in Sec. 3. Numerical experiments involving (RE2), (RE3) or the NavierStokes equation, and illustrating the long-time behavior or the finite speed propagation, are collected in Sec. 4.

2. Basic theory for (RE2). We first introduce a few notations. For any $n \in \mathbb{N}^{*}$, let $H_{p e r}^{n}(\mathcal{Y})$ denote the usual Sobolev space of periodic functions; i.c., $H_{p e r}^{n}(\mathcal{Y})$ is the completion of the space

$$
C_{p e r}^{\infty}(\overline{\mathcal{Y}}):=\left\{v \in C^{\infty}\left(\mathbb{R}^{2}\right) ; v\left(y_{1}+1, y_{2}\right)=v\left(y_{1}, y_{2}\right)=v\left(y_{1}, y_{2}+1\right) \quad \forall y \in \mathbb{R}^{2}\right\}
$$

for the norm

$$
\|v\|_{H_{p e,}^{n}(\mathcal{Y})}:=\left(\sum_{\alpha \in \mathbb{N}^{2} .|\alpha| \leq n}\left\|\partial^{\alpha} v\right\|_{L^{2}(\mathcal{Y})}^{2}\right)^{\frac{1}{2}} .
$$

For the sake of brevity we write $H:=L^{2}(\mathcal{Y}), \tilde{H}:=\left\{v \in H ; \int_{\mathcal{Y}} v(y) d y=0\right\}, V:=$ $H_{\text {per }}^{1}(\mathcal{Y})$ and $\tilde{V}=V \cap \tilde{H}$. $\tilde{V}$ (resp. its dual space $\tilde{V}^{\prime}$ ) is endowed with the norm $\|v\|_{\tilde{V}}=\left(\int_{\mathcal{Y}}|\nabla v|^{2} d y\right)^{\frac{1}{2}}$ (resp. with the dual norm $\|f\|_{\tilde{V}^{\prime}}=\sup _{\|v\|_{\tilde{V}} \leq 1}\langle f, v\rangle_{\tilde{V}^{\prime} \cdot \tilde{V}}$ ). For any $T>0$, let $W(0, T)$ denote the space $\left\{w \in L^{2}(0, T, \tilde{V}), d w / d t \in L^{2}\left(0, T, \tilde{V}^{\prime}\right)\right\}$ endowed with the Hilbertian norm

$$
\|w\|_{W(0, T)}=\left(\|w\|_{L^{2}(0, T, \tilde{V})}^{2}+\left\|\frac{d w}{d t}\right\|_{L^{2}\left(0, T, \bar{V}^{\prime}\right)}^{2}\right)^{\frac{1}{2}} .
$$

Notice that for any $w \in W(0, T), d w / d t \in L^{2}\left(0, T, V^{\prime}\right)$, as well. The following functions appear many times in what follows.

$$
\varphi(x):=\int_{-1}^{x}\left(1-\sigma^{2}\right) d \sigma, \quad S(x):=\frac{1+x}{2} \ln (1+x)+\frac{1-x}{2} \ln (1-x), \quad x \in[-1,1] .
$$

The next result, which combines [18, Thm. 1] and [18, Thm. 2], asserts the existence and uniqueness of a variational solution for (RE2).

ThEOREM 2.1. Let $\bar{\omega}_{0} \in \tilde{H}$ be such that

$$
-1 \leq \bar{\omega}_{0}(y) \leq 1 \text { for a.e. } y \in \mathcal{Y} \text { and }\left|\beta\left(\bar{\omega}_{0}\right)\right|<+\infty \text {. }
$$

Then there exist a time $T>0$ and a unique solution $\bar{\omega} \in W(0, T)$ of the following variational equation

$$
\left\{\begin{array}{l}
\forall v \in L^{2}(0, T, V), \quad \int_{0}^{T}\left\langle\frac{d \bar{\omega}}{d t}, v\right\rangle_{V^{\prime}, V} d t+ \\
\nu \int_{0}^{T} \int_{\mathcal{Y}}\left\{\nabla \varphi(\bar{\omega})-\frac{1}{\nu} \bar{\omega} \nabla \times \bar{\psi}+\beta(\bar{\omega})\left(1-\bar{\omega}^{2}\right)^{2} \nabla \bar{\psi}\right\} \cdot \nabla v d y d t=0
\end{array}\right.
$$

( $\beta$ being defined by $(1.3)$ ), such that

$$
\begin{gathered}
\bar{\omega}(0, \cdot)=\bar{\omega}_{0}, \\
-1 \leq \bar{\omega}(t, y) \leq 1 \text { for a.c. }(t, y) \in[0, T] \times \mathcal{Y}
\end{gathered}
$$

and

$$
\beta(\bar{\omega}(t)) \text { is a (bounded) continuous function on }[0, T] \text {. }
$$


$\bar{\omega}$ is indeed a (weak) solution of (RE2), since (2.2) may be rewritten as

$$
\frac{d \bar{\omega}}{d t}-\nu \operatorname{div}(\nabla \varphi(\bar{\omega}))+\operatorname{div}\left(\bar{\omega} \nabla \times \bar{\psi}-\nu \beta(\bar{\omega})\left(1-\bar{\omega}^{2}\right)^{2} \nabla \bar{\psi}\right)=0 \text { in } L^{2}\left(0, T, V^{\prime}\right)
$$

with $\langle\operatorname{div} u, v\rangle:=-\int_{\mathcal{Y}} u \cdot \nabla v d y$ for $u \in H \times H, v \in V$. Furthermore, it is readily inferred from $(2.2)$ that the kinetic energy $\mathcal{E}(\bar{\omega}):=(1 / 2) \int_{\mathcal{Y}} \bar{\omega} \bar{\psi} d y=(1 / 2)\|\bar{\psi}\|_{\tilde{V}}^{2}=(1 / 2)\|\bar{\omega}\|_{\tilde{V}^{\prime}}^{2}$, is a conserved quantity for the flow of (RE2), whereas the negative mixing entropy $\mathcal{J}(\bar{\omega}):=$ $\int_{\mathcal{Y}} S(\bar{\omega}) d y$ is nonincreasing. (See [18, Prop. 1] for the details.)

REMARK 2.2. Since $W(0, T)$ is continuously embedded in $C([0, T], \tilde{H})$, it follows from (2.4) that $\bar{\omega} \in C\left([0, T], L^{p}(\mathcal{Y})\right)$ for any $p \in[2,+\infty)$ and that $\|\bar{\omega}(t)\|_{L^{\infty}(\mathcal{Y})} \leq 1$ for every $t \in[0, T]$.

Let $\bar{\omega}_{0} \in \tilde{H}$ be such that $(2.1)$ is fulfilled. A map $\bar{\omega}:\left[0, T^{\prime}\right) \times \mathcal{Y} \rightarrow \mathbb{R}$ (with $0<$ $\left.T^{\prime} \leq+\infty\right)$ will be called a solution of (RE2) issuing from $\bar{\omega}_{0}$ if, for any $T \in\left(0, T^{\prime}\right)$, $\bar{\omega}_{\left.\right|_{[0 . T]}} \in W(0, T)$ and $(2.2)-(2.5)$ hold true. Thanks to Theorem 2.1, for any $\bar{\omega}_{0} \in \tilde{H}$ fulfilling (2.1) there exists a unique solution $\bar{\omega}$ of (RE2) issuing from $\bar{\omega}_{0}$ and defined on a right-maximal interval of existence $\left[0, T_{\max }\right.$ ) (with $0<T_{\max } \leq+\infty$ ). The issue whether $T_{\max }=+\infty$ for every $\bar{\omega}_{0} \in \tilde{H}$ fulfilling $(2.1)$ is open at the present time. Nevertheless, we may define a family of subsets $\left(\mathcal{U}_{p, \varepsilon}\right)_{p>2,0<\varepsilon<1}$ of $\tilde{H}$ such that $T_{\text {max }}=+\infty$ whenever $\bar{\omega}_{0} \in \mathcal{U}_{p, \varepsilon}$ for some pair $(p, \varepsilon)$. More precisely, for any $p \in(2,+\infty)$ and any $\varepsilon \in(0,1)$, $\mathcal{U}_{p, \varepsilon}$ is given by

$$
\mathcal{U}_{p, \varepsilon}:=\left\{\bar{\omega} \in \tilde{H} ;\|\bar{\omega}\|_{L^{\infty}(\mathcal{Y})} \leq 1 \text { and } \mathcal{E}(\bar{\omega}) \geq \frac{C(p)}{1-\varepsilon} \mathcal{J}(\bar{\omega})^{1+\frac{2}{p}}\right\},
$$

where

$$
C(p):=\frac{2}{\sqrt{\pi}}\left(\frac{4 p(p-1) \pi^{\frac{3}{2}}}{p-2}\right)^{\frac{2}{p}} .
$$

Due to the conservation of the kinetic energy and the decrease of the negative entropy, $\mathcal{U}_{p, \varepsilon}$ is invariant by the flow of (RE2). It may be seen that its interior in $L^{\infty} \cap \tilde{H}$ (for the $L^{\infty}$ norm) is nonempty. The introduction of $\mathcal{U}_{p . \varepsilon}$ is motivated by the following result $[18]$ :

Theorem 2.3. Let $p>2,0<\varepsilon<1$ and $\bar{\omega}_{0} \in \mathcal{U}_{p, \varepsilon}$. Then

$$
\int_{\mathcal{Y}}\left(1-\bar{\omega}_{0}^{2}\right)^{2}\left|\nabla \psi_{0}\right|^{2} d y \geq \varepsilon\left\|\bar{\omega}_{0}\right\|_{\tilde{V}^{\prime}}^{2} .
$$

As a consequence, $T_{\max }=+\infty$ and $|\beta(\bar{\omega}(t))| \leq\left(3 \varepsilon \mathcal{E}\left(\bar{\omega}_{0}\right)\right)^{-1}$ for all $t \geq 0$. Besides, if $\left(t_{n}\right)_{n \geq 0}$ is any sequence such that $0 \leq t_{n} \nearrow+\infty$, then there exist a subsequence $\left(t_{n_{k}}\right)_{k \geq 0}$ and a function $\tilde{\omega} \in C_{\text {per }}^{\infty}(\overline{\mathcal{Y}})$ (a Gibbs state) fulfilling

$$
\tilde{\omega}=\tanh (-\beta(\tilde{\omega}) \tilde{\psi}+C)
$$

(where $C \in \mathbb{R}$ is some constant and $\tilde{\psi}$ denotes the stream function associated with $\tilde{\omega}$ ), and such that, as $k \rightarrow+\infty$,

$$
\begin{aligned}
\bar{\omega}\left(t_{n_{k}}, \cdot\right) & \rightarrow \tilde{\omega} \operatorname{in} H \\
\beta\left(\bar{\omega}\left(t_{n_{k}}\right)\right) & \rightarrow \beta(\tilde{\omega}) .
\end{aligned}
$$


Assume now that $\omega_{0} \equiv 1$ (hence $\bar{\omega}_{0} \equiv 1$ ) on some open ball $B_{\rho_{0}}\left(y_{0}\right) \subset \mathbb{R}^{2}$. Since the statistical equilibrium state $\tilde{\omega}$ that the system is likely to reach fulfills the Gibbs relation (2.6) (hence $\|\tilde{\omega}\|_{L^{x}(\mathcal{Y})}<1$ ), we infer that the level set $\{\bar{\omega}=1\}$ shrinks and disappears on account of mixing of vorticity over time. Numerical experiments show that this process is gradual. not instantaneous. In other words, the border of the level set $\{\bar{\omega}=1\}$ propagates with a finite speed. Surprisingly enough, this property may be demonstrated by following a method developed by Antontser et al. (see [6]. [1]. [2] and [9]), which rests on some differential inequality for a local energy:

Theorem 2.4. Let $\bar{\omega}_{0}$ be such that $(2.1)$ is fulfilled and $\bar{\omega}_{0}(y)=-1$ (resp. $\left.\bar{\omega}_{0}(y)=1\right)$ for all $y$ in some ball $B_{\rho_{0}}\left(y_{0}\right)$. Let $\bar{\omega}:\left[0, T_{\max }\right) \rightarrow \mathbb{R}$ denote the solution of (RE2) issuing from $\bar{\omega}_{0}$. Then there exist a time $T^{*} \in\left(0 . T_{\text {max }}\right)$ and a continuous function $\rho:\left[0, T^{*}\right] \rightarrow(0,+\infty)$ with $\rho(0)=\rho_{0}$ and such that $\bar{\omega}(t . y)=-1$ (resp. $\left.\bar{\omega}(t, y)=1\right)$ a.e. in $\left\{(t, y) ; 0 \leq t \leq T^{*}, y \in B_{\rho(t)}\left(y_{0}\right)\right\}$.

The interested reader is referred to the next section for a rigorous proof of Theorem 2.4 .

REMARK 2.5. The fact that the disappearance of the level sets $\{\bar{\omega}= \pm 1\}$ is gradual prevents us from using (for small times) the following estimate for $\beta(\bar{\omega})$, which holds true for some constant $C>0$ and for any $\bar{\omega} \in \tilde{H}$ :

$$
|\beta(\bar{\omega})| \leq \frac{C}{\|\bar{\omega}\|_{\tilde{V}}^{2}} \int_{\mathcal{Y}}\left(1-\bar{\omega}^{2}\right)^{-2} d y .
$$

3. Proof of Theorem 2.4. The proof combines the smoothing procedure developed in [18, Proof of Prop. 2] and the trick used by Galiano in [9, Proof of Thm. 2.1] to handle the convection term. (Here, the problematic convection term in (RE2) is $\operatorname{div}(\omega \nabla \times \psi)$.)

We assume that $\omega_{0}(y)=-1$ for all $y \in B_{\rho_{0}}\left(y_{0}\right)$. (The proof is the same when $\omega_{0}=1$ on $B_{\rho_{0}}\left(y_{0}\right)$.) In what follows, we shall write $B_{r}$ for $B_{r}\left(y_{0}\right)$ and we shall omit the bars over $\omega$ and $\psi$, for the sake of simplicity. Fix $T \in\left(0 . T_{\max }\right)$. and set $Q:=(0 . T) \times \mathcal{Y}$. For any function $v=v(t, y)$ in $L^{1}(Q)$ and for any $h \in\left(0, T_{\max }-T\right)$, we set

$$
v^{h}(t, y):=\frac{1}{h} \int_{t}^{t+h} v(\tau, y) d \tau \quad \text { for a.e. }(t, y) \in Q \text {. }
$$

Set $\mathbf{u}=\nabla \times \psi$ and

$$
\begin{aligned}
F(\omega): & =-\operatorname{div}(\omega \mathbf{u})+\nu \beta(\omega) \operatorname{div}\left(\left(1-\omega^{2}\right)^{2} \nabla \psi\right) \\
& =-\nabla \omega \cdot \mathbf{u}-\nu \beta(\omega)\left(4 \omega\left(1-\omega^{2}\right) \nabla \omega \cdot \nabla \psi+\left(1-\omega^{2}\right)^{2} \omega\right) .
\end{aligned}
$$

Then $\mathbf{u} \in C\left([0 . T], W^{1 . p}(\mathcal{Y})^{2}\right)$ for every $1 \leq p<+\infty$ (hence $\mathbf{u} \in C\left([0, T] \times \overline{\mathcal{Y}} \cdot \mathbb{R}^{2}\right)$ ). $F(\omega) \in L^{2}(Q)$ and we readily infer from $(2.2)$ that

$$
\frac{d \omega^{h}}{d t}-\nu \Delta \varphi(\omega)^{h}=F(\omega)^{h} \quad \text { in } L^{2}(Q) .
$$

and that $\varphi(\omega)^{h} \in L^{2}\left(0, T . H_{p c r}^{2}(\mathcal{Y})\right)$. Let

$$
\alpha>\sup _{(t . y) \in[0 . T] \times \overline{\mathcal{Y}}}|\mathbf{u}(t, y)|
$$


and let $\varepsilon \in\left(0, \min \left(\rho_{0}, 2 \alpha T\right)\right)$ be fixed. For every $\rho \in\left[\varepsilon, \rho_{0}\right]$ and every $t \in(0, \varepsilon /(2 \alpha))$ we set

$$
\mathcal{P}=\mathcal{P}(t, \rho):=\left\{(\tau, y) ; 0 \leq \tau \leq t,\left|y-y_{0}\right| \leq \rho-\alpha \tau\right\}
$$

Adding $\nabla \omega^{h} \cdot \mathbf{u}$ to both sides of $(3.1)$, multiplying each term of the equation by $\varphi\left(\omega^{h}\right)$ and integrating on $\mathcal{P}(t, \rho)$ yields

$$
\begin{aligned}
& \iint_{\mathcal{P}}\left(\frac{d \omega^{h}}{d t}+\nabla \omega^{h} \cdot \mathbf{u}\right) \varphi\left(\omega^{h}\right) d y d \tau-\nu \iint_{\mathcal{P}} \Delta \varphi(\omega)^{h} \varphi\left(\omega^{h}\right) d y d \tau \\
& \quad=\iint_{\mathcal{P}}\left(\nabla \omega^{h} \cdot \mathbf{u}+F(\omega)^{h}\right) \varphi\left(\omega^{h}\right) d y d \tau
\end{aligned}
$$

Let us introduce

$$
g(s):=\int_{0}^{s} \sigma\left(\varphi^{-1}\right)^{\prime}(\sigma) d \sigma \quad \text { for } s \in \varphi([-1,1])=\left[0, \frac{4}{3}\right] .
$$

Since $\left(\varphi^{-1}\right)^{\prime}(\sigma) \sim 2^{-1} \sigma^{-\frac{1}{2}}$ as $\sigma \rightarrow 0^{+}$, we get for some constants $C_{1}, C_{2}>0$

$$
C_{1} s^{\frac{3}{2}} \leq g(s) \leq C_{2} s^{\frac{3}{2}} \quad \text { for } 0 \leq s \leq \frac{4}{3}
$$

On the other hand, setting $\theta_{h}=g \circ \varphi \circ \omega^{h}$, we readily get

$$
\frac{d \theta_{h}}{d t}=\varphi\left(\omega^{h}\right) \frac{d \omega^{h}}{d t} \text { and } \nabla \theta_{h}=\varphi\left(\omega^{h}\right) \nabla \omega^{h} .
$$

Hence, applying the divergence theorem to the vector field $\theta_{h}(1, \mathbf{u})$ in the domain $\mathcal{P} \subset$ $\mathbb{R}_{t} \times \mathbb{R}_{y}^{2}$, we get

$$
\begin{aligned}
\iint_{\mathcal{P}}\left(\frac{d \omega^{h}}{d t}+\nabla \omega^{h} \cdot \mathbf{u}\right) \varphi\left(\omega^{h}\right) d y d \tau & =\iint_{\mathcal{P}}\left(\frac{d \theta_{h}}{d t}+\nabla \theta_{h} \cdot \mathbf{u}\right) d y d \tau \\
& =\int_{\partial \mathcal{P}} \theta_{h}\left(n_{t}+\mathbf{u} \cdot n_{x}\right) d \mathcal{H}^{2}
\end{aligned}
$$

where $\left(n_{t}, n_{x}\right)$ denotes the unitary outward normal vector to the boundary $\partial \mathcal{P}$ of $\mathcal{P}$ and $\mathcal{H}^{2}$ denotes the (2-dimensional) Hausdorff measure on $\mathbb{R}^{3}$. Obviously,

$$
\partial \mathcal{P}=\{0\} \times B_{\rho} \cup \bigcup_{0<\tau<t}\{\tau\} \times \partial B_{\rho-\alpha \tau} \cup\{t\} \times B_{\rho-\alpha t}=: \mathcal{P}_{(0)} \cup \partial_{l} \mathcal{P} \cup \mathcal{P}_{(t)}
$$

and

$$
\left(n_{t}, n_{x}\right)= \begin{cases}(-1,0) & \text { on } \mathcal{P}_{(0)} \\ \left(1+\alpha^{2}\right)^{-\frac{1}{2}}\left(\alpha, \frac{x}{|x|}\right) & \text { on } \partial_{l} \mathcal{P} \\ (1,0) & \text { on } \mathcal{P}_{(t)}\end{cases}
$$

Since $n_{t}+\mathbf{u} \cdot n_{x}=\left(1+\alpha^{2}\right)^{-\frac{1}{2}}(\alpha+(\mathbf{u} \cdot x) /|x|)>0$ on $\partial_{l} \mathcal{P}$ (by (3.2)), we get

$$
\iint_{\mathcal{P}}\left(\frac{d \omega^{h}}{d t}+\nabla \omega^{h} \cdot \mathbf{u}\right) \varphi\left(\omega^{h}\right) d y d t \geq \int_{B_{\rho-\alpha t}} \theta_{\left.h\right|_{\tau=t}} d y-\int_{B_{\rho}} \theta_{\left.h\right|_{\tau=0}} d y .
$$

On the other hand, by Green's identity,

$$
\begin{aligned}
& -\iint_{\mathcal{P}} \Delta \varphi(\omega)^{h} \varphi\left(\omega^{h}\right) d y d \tau \\
& \quad=\iint_{\mathcal{P}} \nabla \varphi(\omega)^{h} \cdot \nabla \varphi\left(\omega^{h}\right) d y d \tau-\int_{0}^{t} \int_{\partial B_{\rho-\alpha \tau}}\left(\nabla \varphi(\omega)^{h} \cdot n\right) \varphi\left(\omega^{h}\right) d \mathcal{H}^{1} d \tau,
\end{aligned}
$$


where $n$ denotes the unitary outward normal vector to the circle $\partial B_{\rho-\alpha \tau} \subset \mathbb{R}^{2}$ and $\mathcal{H}^{1}$ denotes the (1-dimensional) Hausdorff measure on $\mathbb{R}^{2}$. An application of the CauchySchwarz inequality yields

$$
\begin{aligned}
& \left|\int_{0}^{t} \int_{\partial B_{\mu-\alpha \tau}}\left(\nabla \varphi(\omega)^{h} \cdot n\right) \varphi\left(\omega^{h}\right) d \mathcal{H}^{1} d \tau\right| \\
& \quad \leq\left(\int_{0}^{t} \int_{\partial B_{p-\alpha \tau}}\left|\nabla \varphi(\omega)^{h}\right|^{2} d \mathcal{H}^{1} d \tau\right)^{\frac{1}{2}}\left(\int_{0}^{t} \int_{\partial B_{p-\alpha \tau}} \varphi\left(\omega^{h}\right)^{2} d \mathcal{H}^{1} d \tau\right)^{\frac{1}{2}} .
\end{aligned}
$$

Let us introduce for all $h \in\left(0, T_{\max }-T\right)$, all $t \in(0,(\varepsilon / 2 \alpha)]$ and all $\rho \in\left[\varepsilon, \rho_{0}\right]$

$$
\begin{aligned}
E_{h}=E_{h}(t, \rho) & :=\iint_{\mathcal{P}}\left|\nabla \varphi\left(\omega^{h}\right)\right|^{2} d y d \tau, \\
b_{h}=b_{h}(t, \rho) & :=\max _{\tau \in[0 . t]} \int_{B_{r_{-\alpha \tau}}}\left|\varphi\left(\omega^{h}\right)\right|^{\frac{3}{2}}(\tau, y) d y .
\end{aligned}
$$

(It is easily seen that $\varphi\left(\omega^{h}\right) \in C\left([0, T], L^{\frac{3}{2}}(\mathcal{Y})\right)$, hence $b_{h}$ is well defined.) We use now the following interpolation-trace result by Diaz-Veron [6, Cor. 2.1]: There exists a constant $C_{3}>0$ such that for every $r \in\left(0, \rho_{0}\right)$ and every $v \in H^{1}\left(B_{r}\right)$

$$
\|v\|_{L^{2}\left(\partial B_{r}\right)} \leq C_{3}\left(\|\nabla v\|_{L^{2}\left(B_{r}\right)}+r^{-\frac{4}{3}}\|v\|_{L_{2}^{\frac{3}{2}\left(B_{r}\right)}}\right)^{\frac{5}{8}}\|v\|_{L_{2}^{\frac{3}{2}\left(B_{r}\right)}}^{\frac{3}{8}} .
$$

Then we get (using also the Hölder inequality)

$$
\begin{aligned}
\int_{0}^{t} & \int_{\partial B_{p-\alpha \tau}} \varphi\left(\omega^{h}\right)^{2} d \mathcal{H}^{1} d \tau \\
\leq & C_{3}^{2} \int_{0}^{t}\left(\left\|\nabla \varphi\left(\omega^{h}\right)\right\|_{L^{2}\left(B_{\rho-\alpha \tau}\right)}+\left(\frac{\varepsilon}{2}\right)^{-\frac{4}{3}}\left\|\varphi\left(\omega^{h}\right)\right\|_{L^{2}\left(B_{\rho-\alpha \tau}\right)}\right)^{\frac{5}{4}} \cdot\left\|\varphi\left(\omega^{h}\right)\right\|_{L^{\frac{3}{2}\left(B_{\rho-\alpha \tau}\right)}}^{\frac{3}{4}} d \tau \\
\leq & C_{3}^{2}\left(\int _ { 0 } ^ { t } \left(\left\|\nabla \varphi\left(\omega^{h}\right)\right\|_{L^{2}\left(B_{\rho-\alpha \tau \tau}\right)}\right.\right. \\
& \left.\left.\quad+\left(\frac{\varepsilon}{2}\right)^{-\frac{t}{3}}\left\|\varphi\left(\omega^{h}\right)\right\|_{L^{\frac{3}{2}\left(B_{p-\alpha \tau}\right)}}\right)^{2} d \tau\right)^{\frac{5}{8}} \cdot\left(\int_{0}^{t}\left\|\varphi\left(\omega^{h}\right)\right\|_{L^{3}\left(B_{\rho-\alpha \tau}\right)}^{2} d \tau\right)^{\frac{3}{8}} \\
& \leq C_{3}^{2}\left(2 E_{h}+2 t\left(\frac{\varepsilon}{2}\right)^{-\frac{8}{3}} b_{h}^{\frac{4}{3}}\right)^{\frac{5}{8}}\left(t b_{h}^{\frac{1}{3}}\right)^{\frac{3}{8}} \\
\leq & C_{3}^{2}\left(2 E_{h}+2 T\left(\frac{\varepsilon}{2}\right)^{-\frac{8}{3}} b_{h}^{\frac{1}{3}} \cdot b_{h}\right)^{\frac{5}{8}} t^{\frac{3}{8}}\left(E_{h}+b_{h}\right)^{\frac{1}{2}} \\
\leq & C_{4}^{2} t^{\frac{3}{8}}\left(E_{h}+b_{h}\right)^{\frac{9}{8}}
\end{aligned}
$$

for some positive constant $C_{4}=C_{4}\left(\varepsilon, T, \rho_{0}\right)$. (Recall that $\varphi$ takes its values in $\left[0, \frac{4}{3}\right]$. hence $b_{h}^{\frac{1}{3}} \leq$ Const.) We infer from (3.3)-(3.7) that for all $h \in\left(0, T_{\max }-T\right)$, all $\rho \in\left[\varepsilon, \rho_{0}\right]$ and all $t \in(0, \varepsilon /(2 \alpha)]$,

$$
\begin{aligned}
& \int_{B_{\rho-\alpha t}} \theta_{\left.h\right|_{\tau=t}} d y-\int_{B_{\ell}} \theta_{\left.h\right|_{\tau=0}} d y+\nu \iint_{\mathcal{P}} \nabla \varphi(\omega)^{h} \cdot \nabla \varphi\left(\omega^{h}\right) d y d \tau \\
& \leq \nu C_{4} t^{\frac{3}{16}}\left(E_{h}+b_{h}\right)^{\frac{9}{16}}\left(\int_{0}^{t} \int_{\partial B_{\rho-\alpha \tau}}\left|\nabla \varphi(\omega)^{h}\right|^{2} d \mathcal{H}^{1} d \tau\right)^{\frac{1}{2}} \\
& \quad+\iint_{\mathcal{P}}\left(\nabla \omega^{h} \cdot \mathbf{u}+F(\omega)^{h}\right) \varphi\left(\omega^{h}\right) d y d \tau .
\end{aligned}
$$

Letting $h \rightarrow 0^{+}$in $(3.8)$, and using the fact that $\omega \in L^{2}(0, T, V) \cap C\left([0, T], L^{\frac{3}{2}}(\mathcal{Y})\right) \cap$ $L^{\infty}(Q)$, we readily get that for all $t \in(0 . \varepsilon /(2 \alpha))$ and for a.e. $\rho \in\left[\varepsilon, \rho_{0}\right]$,

$$
\begin{aligned}
& \int_{B_{p-\alpha t}} \theta_{\left.\right|_{\tau}=t} d y-0+\nu \iint_{\mathcal{P}}|\nabla \varphi(\omega)|^{2} d y d \tau \\
& \leq \nu C_{4} t \frac{3}{16}(E+b)^{\frac{9}{16}}\left(\int_{0}^{t} \int_{\partial B_{\rho-\alpha \tau}}|\nabla \varphi(\omega)|^{2} d \mathcal{H}^{1} d \tau\right)^{\frac{1}{2}} \\
& \quad-\iint_{\mathcal{P}} \nu \beta(\omega)\left(4 \omega\left(1-\omega^{2}\right) \nabla \omega \cdot \nabla \psi+\left(1-\omega^{2}\right)^{2} \omega\right) \varphi(\omega) d y d \tau,
\end{aligned}
$$


where $\theta:=g \circ \varphi \circ \omega$ and

$$
\begin{aligned}
& E=E(t, \rho) \quad:=\iint_{\mathcal{P}}|\nabla \varphi(\omega)|^{2} d y d \tau, \\
& b=b(t, \rho) \quad:=\max _{\tau \in[0, t]} \int_{B_{\rho-\alpha \tau}}|\varphi(\omega)|^{\frac{3}{2}}(\tau, y) d y .
\end{aligned}
$$

(According to Remark 2.2, the map $\tau \mapsto \int_{B_{\rho-\alpha \tau}}|\varphi(\omega)|^{\frac{3}{2}}(\tau, y) d y$ is continuous.) Notice that for every $t \in(0, \varepsilon /(2 \alpha))$

$$
\frac{\partial E}{\partial \rho}=\int_{0}^{t} \int_{\partial B_{\rho-\alpha \tau}}|\nabla \varphi(\omega)|^{2} d \mathcal{H}^{1} d \tau \quad \text { for a.e. } \rho \in\left[\varepsilon, \rho_{0}\right] .
$$

On the other hand,

$$
\begin{aligned}
& \left|\iint_{\mathcal{P}} 4 \nu \beta(\omega) \omega\left(1-\omega^{2}\right) \nabla \omega \cdot \nabla \psi \varphi(\omega) d y d \tau\right| \\
& \quad=4 \nu\left|\iint_{\mathcal{P}} \beta(\omega) \omega \varphi(\omega) \nabla \varphi(\omega) \cdot \nabla \psi d y d \tau\right| \\
& \quad \leq \nu\left(\iint_{\mathcal{P}}|\nabla \varphi(\omega)|^{2} d y d \tau\right)^{\frac{1}{2}} \cdot\left(\iint_{\mathcal{P}} 16 \beta(\omega)^{2} \omega^{2} \varphi(\omega)^{2}|\nabla \psi|^{2} d y d \tau\right)^{\frac{1}{2}}
\end{aligned}
$$

by the Cauchy-Schwarz inequality. Noticing that for any $(\tau, y) \in(0, T) \times \overline{\mathcal{Y}}$

$$
|\nabla \psi(\tau, y)|=|\mathbf{u}(\tau, y)|<\alpha
$$

we get

$$
\begin{aligned}
& \left|\iint_{\mathcal{P}} 4 \nu \beta(\omega) \omega\left(1-\omega^{2}\right) \nabla \omega \cdot \nabla \psi \varphi(\omega) d y d \tau\right| \\
& \quad \leq \frac{\nu}{2}\left\{\frac{E}{2}+32\|\beta\|_{L^{\infty}(0, T)}^{2} \alpha^{2} \iint_{\mathcal{P}} \varphi(\omega)^{\frac{1}{2}} \varphi(\omega)^{\frac{3}{2}} d y d \tau\right\} \\
& \quad \leq \frac{\nu}{4} E+C_{5} t b
\end{aligned}
$$

for $C_{5}:=(32 / \sqrt{3}) \nu \alpha^{2}\|\beta\|_{L^{\infty}(0, T)}^{2}$. Finally, observing that $(1+\omega)^{2}=3 \varphi(\omega)(2-\omega)^{-1}$ we obtain

$$
\begin{aligned}
\left|\iint_{\mathcal{P}} \nu \beta(\omega)\left(1-\omega^{2}\right)^{2} \omega \varphi(\omega) d y d \tau\right| & \leq 3 \nu\|\beta\|_{L^{\infty}(0, T)} \iint_{\mathcal{P}} \frac{(1-\omega)^{2}|\omega|}{2-\omega} \varphi(\omega)^{2} d y d \tau \\
& \leq C_{6} t b,
\end{aligned}
$$

for some constant $C_{6}>0$. Combining (3.4) and (3.9)-(3.13) we get

$$
C_{1} \int_{B_{\rho-\alpha t}}|\varphi(\omega)|^{\frac{3}{2}}(t, y) d y+\nu E \leq \frac{\nu}{4} E+\left(C_{5}+C_{6}\right) t b+\nu C_{4} t^{\frac{3}{16}}(E+b)^{\frac{9}{16}}\left(\frac{\partial E}{\partial \rho}\right)^{\frac{1}{2}} .
$$

Since $E, b$ and $\partial E / \partial \rho$ are nondecreasing functions of $t$, we get

$$
C_{1} b+\nu E \leq 2\left\{\frac{\nu}{4} E+\left(C_{5}+C_{6}\right) t b+\nu C_{4} t^{\frac{3}{16}}(E+b)^{\frac{9}{16}}\left(\frac{\partial E}{\partial \rho}\right)^{\frac{1}{2}}\right\}
$$

hence there exist $t_{0} \in(0, \varepsilon /(2 \alpha))$ and $C_{7}>0$ such that for all $t \in\left(0, t_{0}\right)$, it holds that

$$
E^{\frac{7}{8}} \leq(E+b)^{\frac{7}{8}} \leq C_{7} t^{\frac{3}{8}} \frac{\partial E}{\partial \rho} \quad \text { for a.e. } \rho \in\left[\varepsilon, \rho_{0}\right] .
$$

Fix $t \in\left(0, t_{0}\right)$. Let $\rho^{*}$ denote the last $\rho \in\left[\varepsilon, \rho_{0}\right]$ for which $E(t, \rho)=0$. (We set $\rho^{*}=\varepsilon$ if $E(t, \rho)>0$ for all $\rho \in\left[\varepsilon, \rho_{0}\right]$.) Assume $\rho^{*}<\rho_{0}$ and pick any $\bar{\rho} \in\left(\rho^{*}, \rho_{0}\right)$. Integrating (3.14) w.r.t. $\rho$ on $\left(\bar{\rho}, \rho_{0}\right)$, we obtain

$$
\rho_{0}-\bar{\rho} \leq 8 C_{7} t^{\frac{3}{8}}\left(E^{\frac{1}{8}}\left(t, \rho_{0}\right)-E^{\frac{1}{8}}(t, \bar{\rho})\right) \leq 8 C_{7} E^{\frac{1}{8}}\left(t_{0}, \rho_{0}\right) t^{\frac{3}{8}},
$$


hence $\bar{\rho} \geq \rho_{0}-C_{8} t^{\frac{3}{8}}$ for some constant $C_{8}>0$. In any case $\rho^{*} \geq \rho_{0}-C_{8} t^{\frac{3}{8}}$. Let $t_{1}=\min \left(t_{0},\left(\left(\rho_{0}-\varepsilon\right) / C_{8}\right)^{\frac{8}{3}}\right)$. Then for any $t \in\left(0, t_{1}\right), E(t, \cdot)=0$ in $\left[\varepsilon, \rho_{0}-C_{8} t^{\frac{3}{8}}\right]$ (and the same property holds true for $\partial E / \partial \rho$ and then for $b(t, \cdot)$ by $(3.14))$. It follows that

$$
\int_{0}^{t_{1}}\left(\int_{\left.B\left(\rho_{0}\right)-c_{8}+\frac{3}{8}\right)-\ldots t}|\varphi(\omega)|^{\frac{3}{2}}(t, y) d y\right) d t \leq \int_{0}^{t_{1}} b\left(t, \rho_{0}-C_{8} t^{\frac{3}{8}}\right) d t=0 .
$$

Finally, $\varphi(\omega)=0 \Longleftrightarrow \omega=-1$, so the proof of Theorem 2.4 is completed by setting $\rho(t):=\rho_{0}-C_{8} t^{\frac{3}{8}}-\alpha t$ and choosing $T^{*}<t_{1}$ in such a wav that $\rho\left(T^{*}\right)>0$.

4. Numerical experiments. In the following numerical simulations the domain $\mathcal{Y}$ is $(-\pi, \pi)^{2}$ instead of $\left(-\frac{1}{2}, \frac{1}{2}\right)^{2}$.

4.1. Initial states. Four initial data will be considered here.

- In the first set of numerical experiments (corresponding to Figures 1, 2, and 5), the initial vorticity $\omega_{0}$ is defined by

$$
\omega_{0}(y)= \begin{cases}1 & \text { in the squares }(-\pi, 0) \times(0, \pi) \text { and }(0, \pi) \times(-\pi, 0) . \\ -1 & \text { clsewhere; }\end{cases}
$$

- In the second one (Figures 3, 4, 6, and 7), $\omega_{0}$ is defined by

$$
\omega_{0}(y)=\left\{\begin{array}{lll}
a_{1} & \text { in the ellipse } & y_{1}^{2} / r_{1}^{2}+y_{2}^{2} / r_{2}^{2} \leq 1 \\
a_{2} & \text { in the annulus } & r_{3}^{2} \leq y_{1}^{2}+y_{2}^{2} \leq r_{4}^{2} \\
0 & \text { elsewhere. }
\end{array}\right.
$$

Since the mean value of $\omega_{0}$ on $\mathcal{Y}$ must be zero the parameters $a_{1}, a_{2}, r_{1}, r_{2}, r_{3}, r_{4}$ fulfill $a_{1} r_{1} r_{2}=a_{2}\left(r_{3}^{2}-r_{4}^{2}\right)$. We shall take $a_{2}=2 \pi, r_{1}=0.5, r_{2}=0.3, r_{3}=$ $0.65, r_{4}=1$;

- In the third set of numerical experiments (Figures 8, 9, and 10), $\omega_{0}$ is defined by

$$
\omega_{0}(y)=\left\{\begin{array}{lll}
1 & \text { in the disk } & y_{1}^{2}+y_{2}^{2} \leq r_{0}^{2}, \\
-1 & \text { in the annulus } & r_{0}^{2} \leq y_{1}^{2}+y_{2}^{2} \leq 4 r_{0}^{2} \\
1 & \text { in the annulus } & 4 r_{0}^{2} \leq y_{1}^{2}+y_{2}^{2} \leq 9 r_{0}^{2} \\
-1 & \text { in the annulus } & 9 r_{0}^{2} \leq y_{1}^{2}+y_{2}^{2} \leq r_{1}^{2} \\
0 & \text { elsewhere. }
\end{array}\right.
$$

The parameters $r_{0}, r_{1}$ have to satisfy $r_{1}=r_{0} \sqrt{12}$. We shall take $r_{0}=0.25$ :

- In the last one (Figures 11 and 12), $\omega_{0}$ is defined by

$$
\omega_{0}(y)= \begin{cases}1 & \text { in the disk } y_{1}^{2}+y_{2}^{2} \leq r_{0}^{2} . \\ -1 & \text { elsewhere. }\end{cases}
$$

The only possible value for $r_{0}$ is $r_{0}=\sqrt{2 \pi}$.

Notice that the two first initial data are not axially symmetric, so we do not need to destabilize the system: The mixing process leads quickly enough to the final structure. Notice also that $\left|\bar{\omega}_{0}\right|=1$ a.e. (hence $\left|\beta\left(\bar{\omega}_{0}\right)\right|=+\infty$ ) in the first and in the last experiments. To overcome this problem, the simulation of (RE2) for these initial data is preceded by a Navier-Stokes simulation over a (very) short-time interval (a few iterations), in order to produce a bit of mixing. 
4.2. Numerical features. Due to the periodic boundary conditions, we use for the spatial discretization a classical pseudo-spectral method. The time discretization scheme is a third order Adams-Bashforth scheme. The same numerical treatment is applied both to Navier-Stokes equations and to the relaxation equations $(R E 2)$ and $(R E 3)$. The additional nonlinear terms in the relaxation equations are evaluated by means of pseudo-spectral transform methods, the aliasing error being removed by using a discrete transform with $3 N / 2$ points, where $N$ denotes the mesh points number. Since the typical velocity and width for the observed vorticity are unitary, $R e=1 / \nu$ is the actual Reynolds number in Navier-Stokes equations. Attention has now to be paid to the (degenerate) viscosity term in the relaxation equations. We know that it can vanish at some places, and we may wonder if this causes numerical instabilities. Actually, this does not occur here: The variable coefficient (namely, $\left(1-\bar{\omega}^{2}\right)$ for (RE2)) vanishes only where the flow has no tendency to develop small-scale oscillations. Elsewhere, oscillations are smoothed out thanks to the viscosity term. Nevertheless, numerical filters (see [22]) will be applied to the solution in order to properly handle the initial vorticity discontinuity. The value of the coefficient $\nu$ (in (RE2) or (RE3)) is taken as in [17]. Recall that beyond some value $\left(10^{-2}\right.$ or $10^{-3}$ in our examples), the numerical results for the relaxation equations are quite indistinguishable and very similar to those obtained for the Navier-Stokes equation. We shall take the greatest value of the coefficient $\nu$ for which this property holds true. The spatial resolution is adapted to the above choice of parameters. The Navier-Stokes simulations with $R e=5000$ require 512 grid points in each direction. whereas for the relaxation equations (RE2), (RE3) (with a larger coefficient $\nu$ ), only 128 grid points (in each direction) are needed. The corresponding time step which guarantees numerical stability is $\Delta t=0.001$.

4.3. Description of the results and comments. The evolution of the flow is represented by successive snapshots of the vorticity field at different times, in $2 D$ for the two first experiments and in $3 D$ for the third experiment. In each simulation we observe that, after an intricate mixing process, the system stabilizes into a final equilibrium state which depends only on the initial datum. In the first numerical experiment (four squares), the system reaches a steady $y_{2}$-independent structure which is a parallel shear layer (Figures 1 and 2). This equilibrium state proves to be almost completely homogeneous in the $y_{2}$-direction and indicates a great mixing for the vorticity.

The second experiment is concerned with the formation of a tripolar coherent structure. This example has been already studied both numerically ([3], [4]. [13]) and experimentally ([7], [23]). In each simulation, we observe that the final state has a steady configuration in a rotating reference frame (see Figures 3 and 4).

In these two experiments we observe that the results obtained with the relaxation equations ((RE2) or (RE3)) are in complete agreement with those obtained with the Navier-Stokes equation. However, a loss of about $50 \%$ of the kinetic energy and a diffusion of the final structure are observed in Navier-Stokes direct simulations, whereas the overall loss of energy does not exceed $6 \%$ for the relaxation equations. The final state in each simulation may be analyzed by representing each point of the computation grid on a scatter plot with abscissa $\psi$ and ordinate $w$. Recall that for a stationary solution of the Euler equation all these points have to collapse on a curve or a set of curves. When 
$\omega_{0}= \pm 1$, it follows from (2.6) that a straight line has to be obtained when plotting $\ln \frac{1-\omega}{1+\omega}$ versus $\psi$. The agreement is very good inside the region of significant vorticity mixing (Fig. 5). In the case of the tripolar structure (Figures 6 and 7), the graph $\omega$ versus $\psi$ may be analyzed as the superposition of two curves. It indicates that there is a maximum entropy state not in the whole domain $\mathcal{Y}$, but only in a bounded subdomain. Indeed, if we restrict ourselves to the tripolar structure, the branch containing the zero level disappears. In this experiment. the computed final structure results both from the tendency of the system to maximize the entropy functional and from the dynamics, which tends to freeze the system into some local Gibbs state.

The aim of the third experiment is to compare numerical simulations using (RE2) (two levels, \pm 1 ), (RE3) (three levels, $-1,0,1$ ) or the Navier-Stokes equation. The final state obtained with (RE2) does not coincide with the one provided by (RE3) (or by the Navier-Stokes equation). (See Figures 8, 9, and 10.) This is not surprising. since in the simulation using (RE2) the level 0 is viewed as a microscopic mixing of the levels -1 and 1 , whereas for this initial datum there is actually no mixing of vorticity in $\mathcal{Y}$. Therefore, only the numerical integration of (RE3) is meaningful in this case.

In the last experiment we are concerned with the finite speed propagation for the extremal values of the vorticity. Here, the initial vorticity is assumed to take the value 1 on a ball centered at the origin, and the value -1 elsewhere. Successive snapshots of the level set $\left\{\omega=1\left( \pm 10^{-3}\right)\right\}$ are provided both for the relaxation equation (RE2) (Fig. 11) and for the Navier-Stokes equation at high Reynolds number (Fig. 12). For the relaxation equation the set $\{\bar{\omega}=1\}$ has a "core-halo" structure. The core is a small white spot centered at the origin. It is surrounded by a low density halo, with an empty interior. The white spot gradually shrinks and disappears at $t=T^{*}=0.25 \mathrm{~s}$. This is in complete agreement with the prediction given in Theorem 2.4, namely the fact that the origin belongs to the interior of the set $\{\bar{\omega}=1\}$ for small positive times. It means that the value -1 of the microscopic vorticity $(\omega)$ propagates with a finite speed in $B_{\sqrt{2 \pi}}(0)$. This does not occur with the Navier-Stokes equation, even at high Reynolds number. Indeed. the level set $\{\omega=1\}$ proves to have an empty interior for $t \geq 0.1 \mathrm{~s}$ in Navier-Stokes simulation. It means that the mixing process occurs everywhere (even in the core of the patch) from the begiming. Notice that the set $\{\omega=1\}$ in Navier-Stokes simulation turns out to be empty from $t=0.1 \mathrm{~s}$ if we pick the same coefficient $\nu$ as the one in the simulation of (RE2) (namely $\nu=10^{-2}$, instcad of $\nu=1 / 2000=5.10^{-4}$ ).

4.4. Conclusion. The relaxation equations can describe accurately the large scale motion without having to explicitly handle the small scales. The statistical mechanics take into account the small scale "chaos" produced by the dymamics of Euler equations. In the case of an initial condition with $n$ vorticity levels, the statistical theory predicts that after some complex transient mixing, the flow tends towards a steady state which is characterized by a simple relationship between vorticity and stream function. The relationship $w=f(\psi)$ for the final state provided by the different numerical simulations is in very good agreement with the Gibbs state relationship predicted by the theory, in regions where significant mixing between the initial vorticity levels occurs. Besides, we observe through these different examples a striking agreement between the computed flows for (REn) and the flows obtained from high-Reynolds number Navier-Stokes direct 

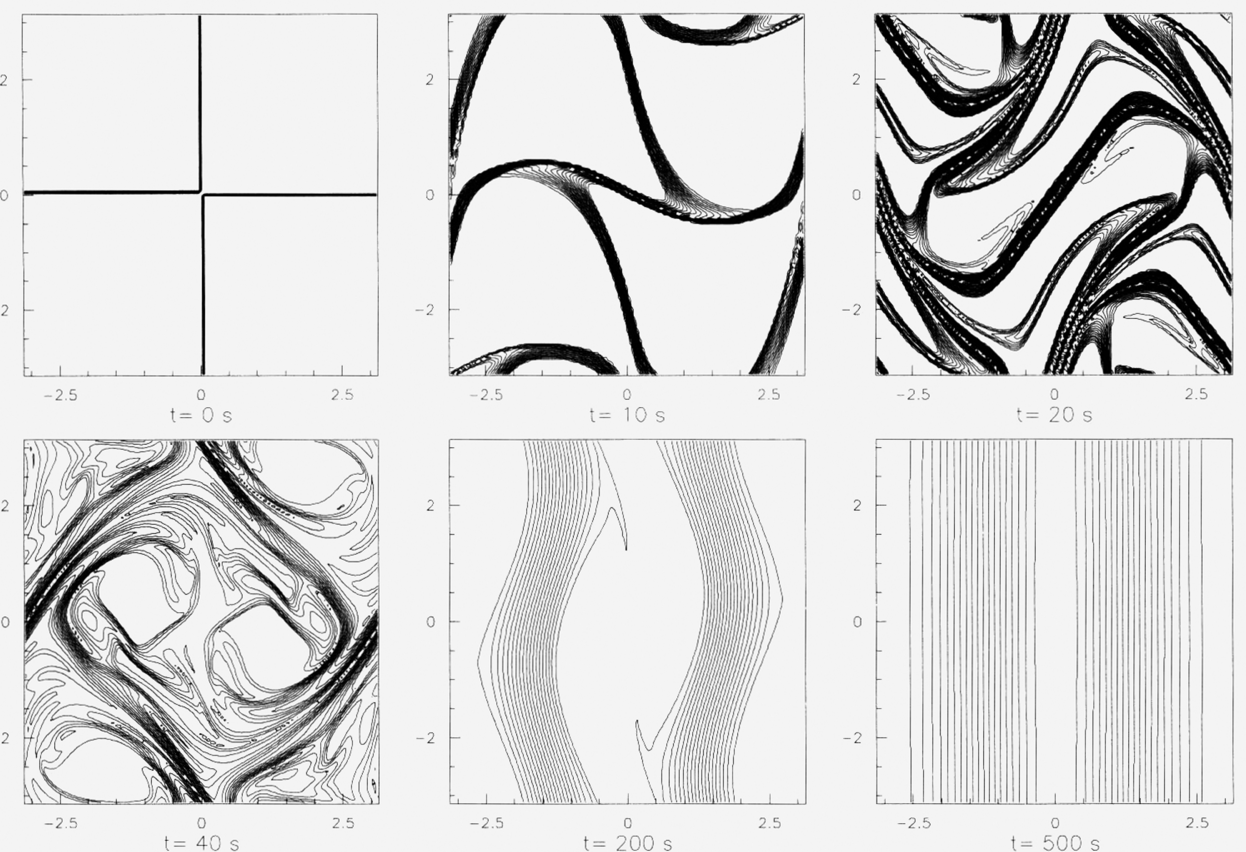

FIG. 1. Successive snapshots of the vorticity field; (RE2), $\nu=10^{-3}$
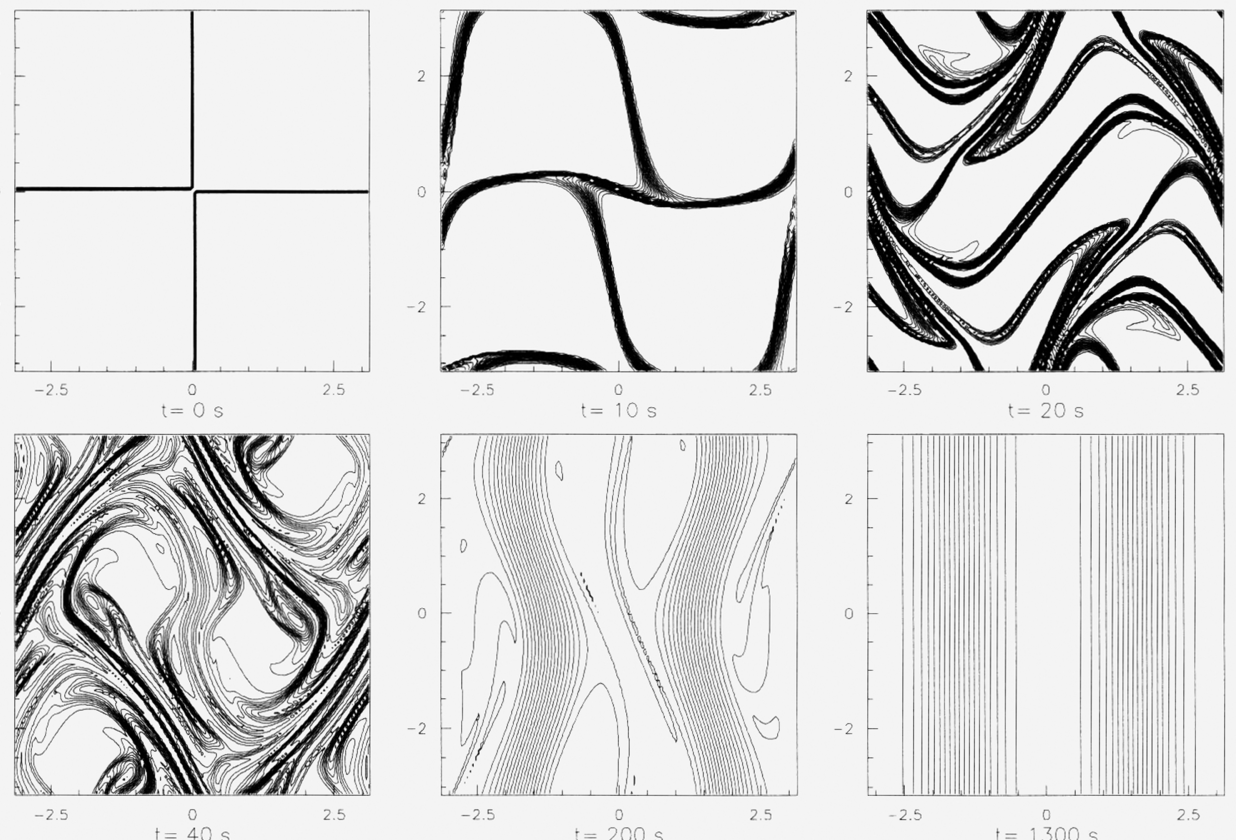

FIG. 2. Successive snapshots of the vorticity field; Navier-Stokes, $R e=5000$ 

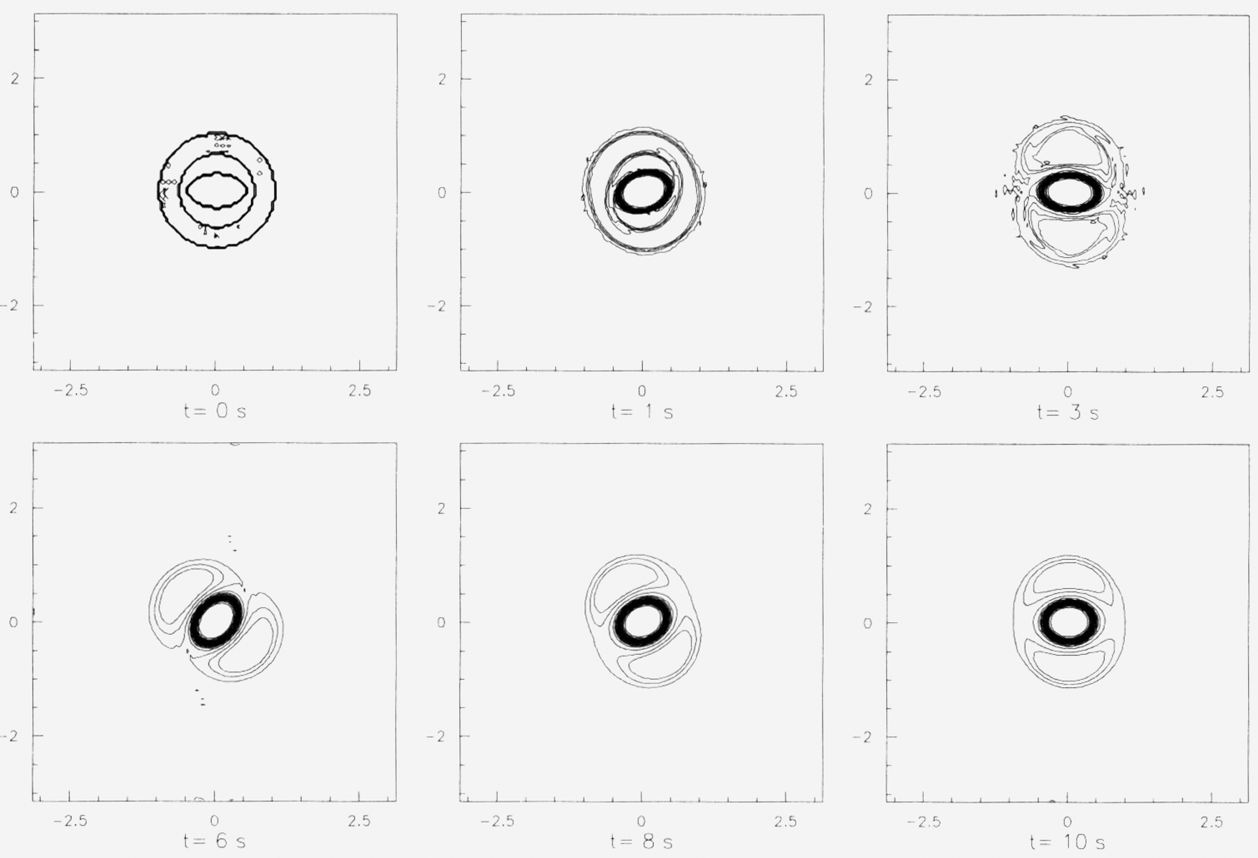

FIG. 3. Successive snapshots of the vorticity field; (RE3), $\nu=10^{-2}$
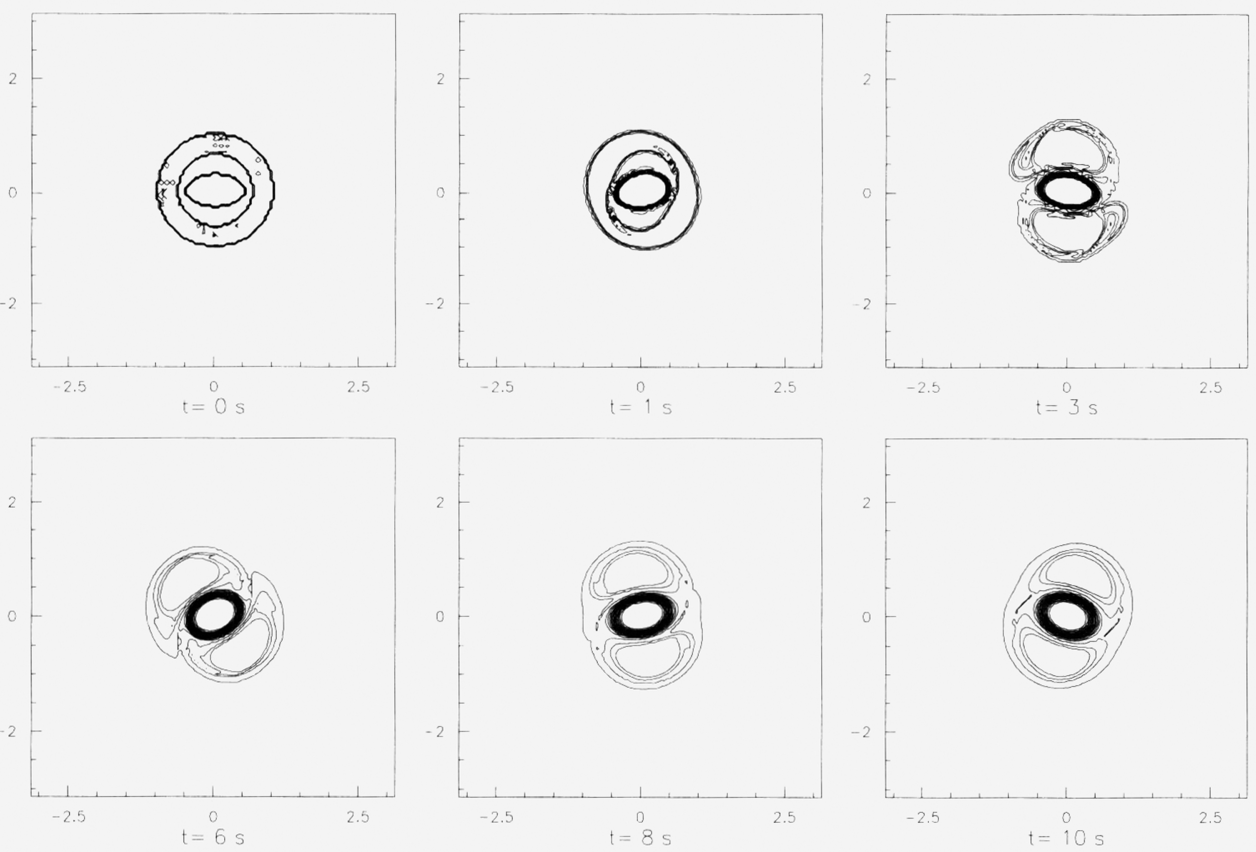

FIG. 4. Successive snapshots of the vorticity field; Navier-Stokes, $R e=5000$ 


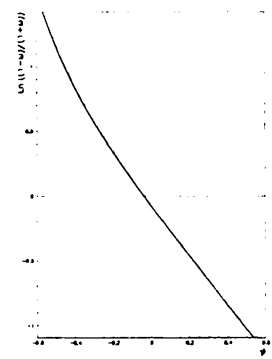

FIG. 5. $\ln \frac{1-\omega}{1+\omega}$ versus $\psi ;(\mathrm{RE} 2), \nu=10^{-3}$

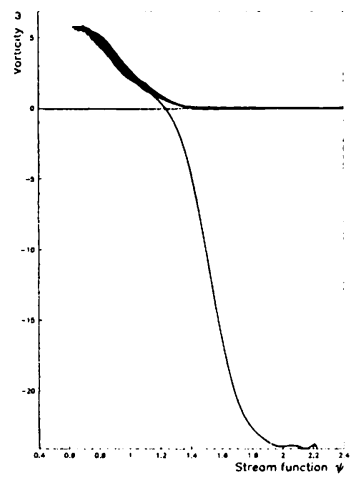

FIG. 6. Vorticity versus stream function; (RE3), $\nu=10^{-2}$

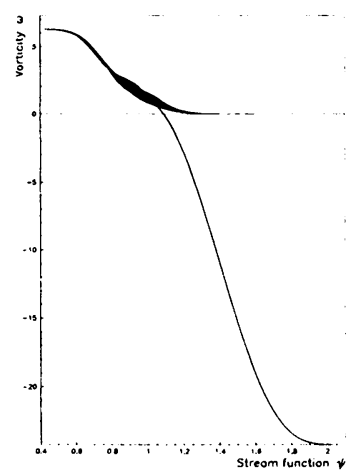

FIG. 7. Vorticity versus stream function; Navier-Stokes, $R e=5000$

simulations. The acceptable value for the coefficient $\nu$ in the variable viscosity term in $(\mathrm{REn})$ is larger than the viscosity coefficient needed for Navier-Stokes computations in 

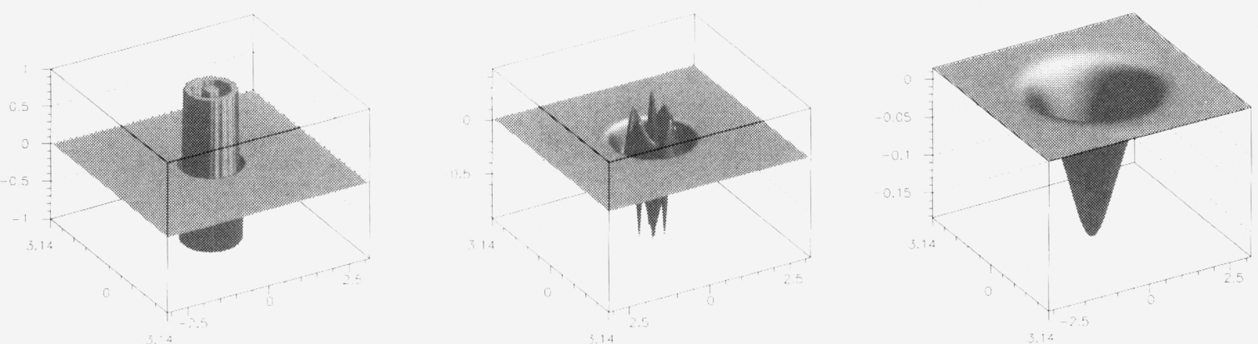

FIG. 8. Evolution of the vorticity field in 3D; (RE2), $\nu=10^{-3}$
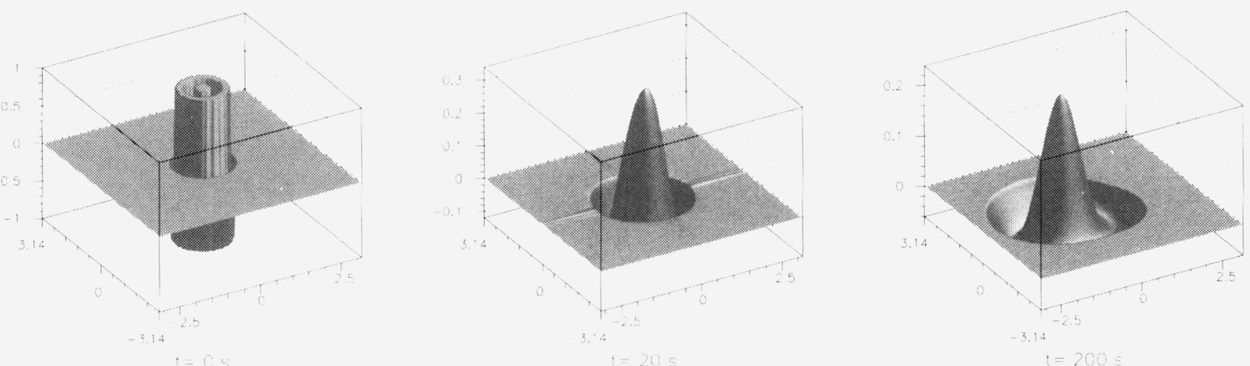

FIG. 9. Evolution of the vorticity field in 3D; (RE3), $\nu=10^{-3}$
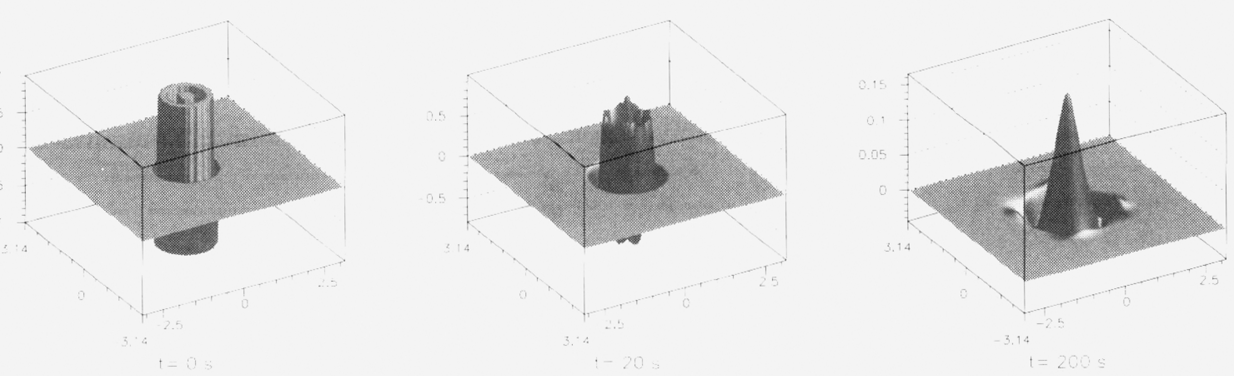

FIG. 10. Evolution of the vorticity field in 3D; Navier-Stokes, $R e=6000$

order to provide the same final coherent structure. It allows us to win computational time through the choice of the spatial resolution and the time step.

The computations have been performed on the CRAY2 of the C.C.V.R. at Palaiseau and on a DEC Alpha 21162 workstation at the Laboratory of Numerical Analysis of the University of Lyon 1.

Acknowledgments. The authors wish to acknowledge G. Galiano for fruitful discussions and L.-H. Rosier in offering judicious advice for the numerical simulations and the graphics. 


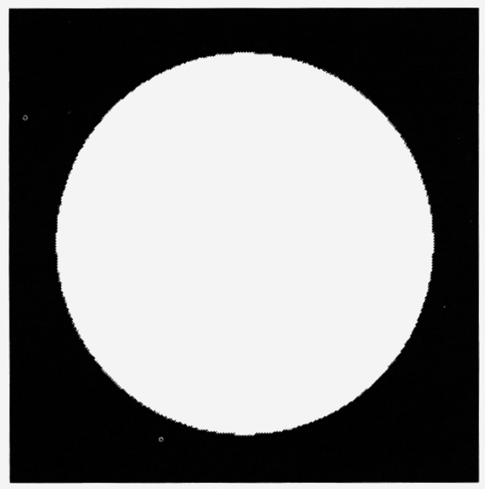

$\mathrm{t}=\mathbf{0 s}$

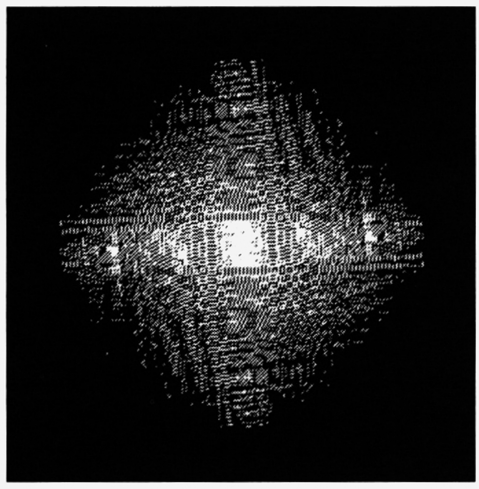

$t=0.2 \mathrm{~s}$

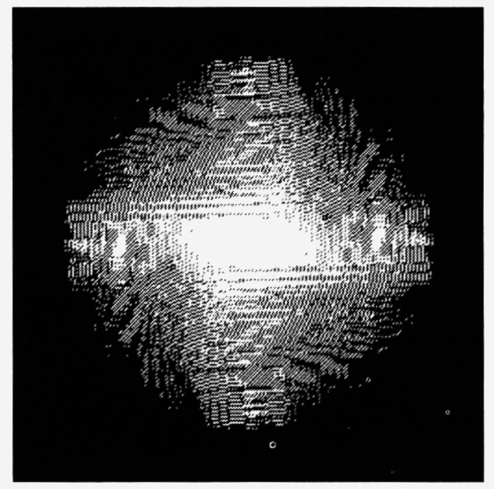

$t=0.1 s$

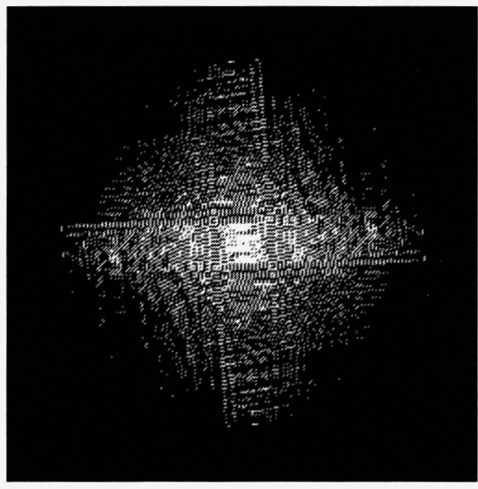

$t=0.25 \mathrm{~s}$

FIG. 11. Successive snapshots of the level set $\{\omega=1\} ;\left(\right.$ RE2), $\nu=10^{-2}$ 


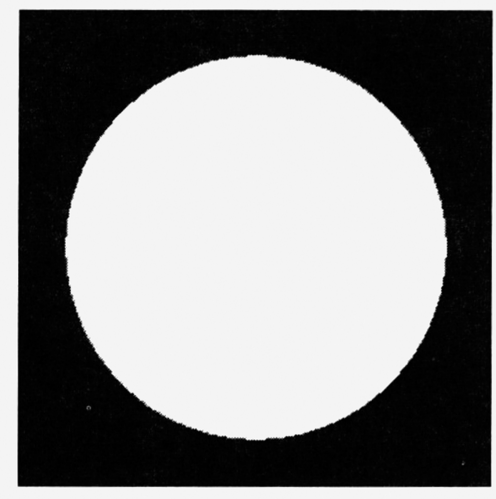

$t=0 \mathrm{~s}$

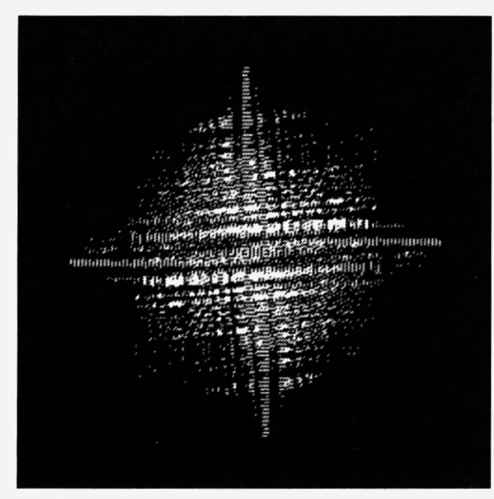

$t=0.2 \mathrm{~s}$

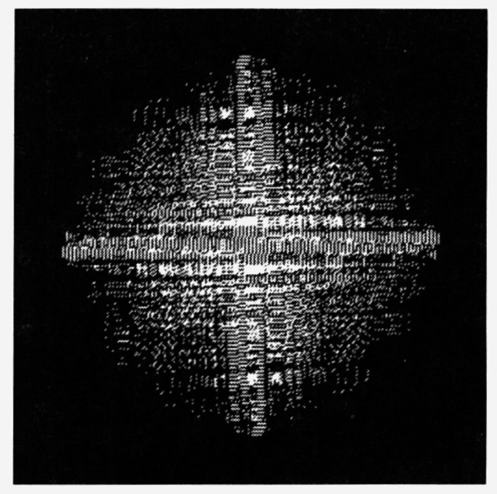

$t=0.1 \mathrm{~s}$

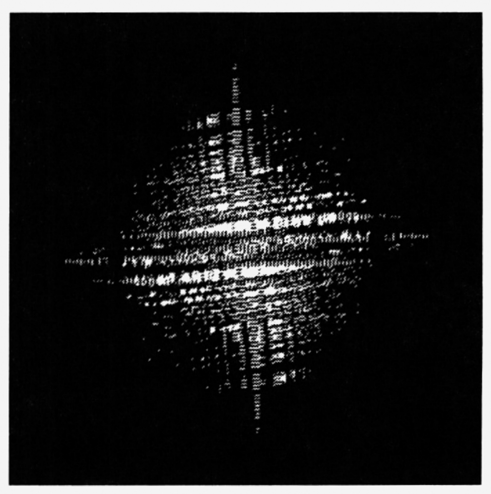

$t=0.25 \mathrm{~s}$

FIG. 12. Successive snapshots of the level set $\{\omega=1\}$; Navier-Stokes, $R e=2000$ 


\section{REFERENCES}

[1] S. N. Antontsev and J. I. Diaz, New results on the localization of solutions of nonlinear elliptic and parabolic equations that are obtained by the energy method, Soviet Math. Dokl. 38 (1989), 535-539.

[2] S. N. Antontsev and J. I. Diaz, Space and time localization in the flow of two immiscible fluids through a porous medium: energy methods applied to systems, Nonlinear Anal., TMA 16 (1991), 299-313.

[3] X. J. Carton, G. R. Flierl, L. M. Polvani, The generation of tripoles from unstable axisymmetric isolated vortex structure, Europhys. Lett. 9 (1989), 339-344.

[4] X. J. Carton, B. Legras, The life-cycle of tripoles in two-dimensional incompressible flows, J. Fluid Mech. 267 (1994), 53-82.

[5] J. I. Diaz and G. Galiano, On the Boussinesq system with non linear thermal diffusion, Nonlinear Anal., TMA 30 (1997), 3255-3263.

[6] J. I. Diaz and L. Veron, Local vanishing properties of solutions of elliptic and parabolic quasilinear equations, Trans. Am. Math. Soc. 290 (1985), 787-814.

[7] J. B. Flor, Coherent vortex structures in stratified fluids, Thesis, Eindhoven, 1994.

[8] G. Gagneux and M. Madaune-Tort, Analyse mathématique de modèles non linéaires de l'ingénierie pétrolière, Mathématiques \& Applications, 22, Springer, 1996.

[9] G. Galiano, Spatial and time localization of solutions of the Boussinesq system with nonlinear thermal diffusion, Nonlinear Anal., TMA 42 (2000), 423-438.

[10] A. Mikelic and R. Robert, On the equations describing a relaxation towards a statistical equilibrium state in the two-dimensional perfect fluid dynamics, SIAM J. Math. Anal. 29 (1998), 1238-1255.

[11] J. Miller, Statistical mechanics of Euler equations in two dimensions, Phys. Rev. Lett. 65 (1990), p. 2137.

[12] J. Miller, P. Weichman and M. C. Cross, Statistical mechanics, Euler's equation, and Jupiter's Red Spot, Phys. Rev. A 45 (1992), 2328-2359.

[13] Y. G. Morel, X. J. Carton, Multipolar vortices in two-dimensional incompressible flows, J. Fluid Mech. 267 (1994), 23-51.

[14] R. Robert, A maximum entropy principle for two-dimensional Euler equations, J. Statist. Phys. 65 (1991), 531-553.

[15] R. Robert and J. Sommeria, Statistical equilibrium states for two-dimensional flows, J. Fluid Mech. 229 (1991), 291-310.

[16] R. Robert and J. Sommeria, Relaxation towards a statistical equilibrium state in two-dimensional perfect fluid dynamics, Phys. Rev. Lett. 69 (1992), 2276-2279.

[17] R. Robert and C. Rosier, The modeling of small scales in two-dimensional turbulent flows: a statistical mechanics approach, J. Stat. Phys. 86 (1997), 481-515.

[18] C. Rosier and L. Rosier, Well-posedness of a degenerate parabolic equation issuing from twodimensional perfect fluid dynamics, Applic. Anal. 75 (2000), 441-465.

[19] J. Sommeria, C. Staquet and R. Robert, Final equilibrium state of a two-dimensional shear layer, J. Fluid Mech. 233 (1991), 661-689.

[20] B. Turkington and N. Whitaker, Statistical equilibrium computations of coherent structures in turbulent shear layers, SIAM J. Sci. Comput. 17 (1996), 1414-1433.

[21] B. Turkington and N. Whitaker, Maximum entropy states for rotating vortex patches, Phys. Fluids 6 (1994), 3963-3973.

[22] H. Vandeven, Family of spectral filters for discontinuous problems, Ecole polytechnique, Internal report No. 159, 1987.

[23] G. J. F. Van Heijst, R. C. Kloosterziel and C. W. M. Williams, Laboratory experiments on the tripolar vortex in a rotating fluid, J. Fluid Mech. 225 (1991), 301-331. 